\title{
A Novel Care Model for Neonatal Intestinal Failure Patients Is Associated With Cost Savings and Improved Outcomes
}

\author{
Erin Johnson ${ }^{\mathrm{a}}$, Anna Ermarth ${ }^{\mathrm{a}}$, Mark Deneau ${ }^{\mathrm{a}, \mathrm{b}}$
}

\begin{abstract}
Background: Neonates with intestinal failure (IF) have prolonged admissions in the neonatal intensive care unit (NICU) and require lifelong follow-up with gastroenterology (GE) as outpatients. Inpatient management of these patients typically relies on many rotating practitioners and currently discharge criteria do not exist. We sought to create standardized discharge criteria with a continuity care model for neonatal IF patients.
\end{abstract}

Methods: Inpatient care was streamlined to two GE physicians with weekly consultations. We implemented standardized discharge goals for both enteral and total parental nutrition (TPN) by: 1) Enteral feedings of at least $5 \mathrm{~mL} / \mathrm{h}$ were tolerated; 2) Stable central venous access was intact; 3) TPN was cycled to $20 \mathrm{~h}$ /day or less; and 4) No other medical issues required NICU admission. Patient records were reviewed after 18 months of implementing standardized discharge criteria and we compared their outcomes to a historical cohort of IF patients.

Results: Optimal discharge criteria were met in 12 patients and a cohort of 26 historical patients was used for comparison. Patients in optimal versus historical groups had similar baseline characteristics (medians, all $\mathrm{P}$ values $=$ non-significant $(\mathrm{NS}))$ : gestational age $(36$ vs. 35 weeks), birth weight (1,990 vs. 2,076 g), birth length (45 vs. $44 \mathrm{~cm}$ ), and small bowel length after definitive surgery (63 vs. 55 $\mathrm{cm}$ ). Compared to the historical group, the optimal cohort was discharged earlier (median length of stay 69 vs. 126 days, $\mathrm{P}<0.01$ ), with a reduced total stay of 684 NICU days, fewer central line-associated bloodstream infections (CLABSIs) (4 vs. 10 per 1,000 patient days, $\mathrm{P}$ $=0.04$ ), and had fewer readmissions ( 7 vs. 17 per 1,000 patient days, $\mathrm{P}<0.01)$, respectively.

Conclusions: Concentrating the care of IF patients to a GE team invested in long-term care, while implementing safe discharge criteria, resulted in a dramatic length of stay reduction with fewer CLABSIs and readmissions compared to historical management. At approxi-

Manuscript submitted January 31, 2019, accepted March 5, 2019

aDepartment of Pediatrics, Division of Gastroenterology, Hepatology and Nutrition, University of Utah, Salt Lake City, UT, USA

${ }^{b}$ Corresponding Author: Mark Deneau, Division of Gastroenterology, Hepatology and Nutrition, University of Utah, 81 N. Mario Capecchi Dr. Salt Lake City, UT 84113, USA. Email: mark.deneau@hsc.utah.edu

doi: https://doi.org/10.14740/gr1149 mately 4,000 dollars per day in NICU hospital charges, this program saved over 2.7 million dollars in care costs while allowing families and their infants more time at home. The safety and applicability of the optimal discharge criteria presented here should be studied further. Similar programs may be effective at other large NICUs.

Keywords: Care model; Neonatal intestinal failure patients; Discharge criteria; Cost savings; Improved outcomes

\section{Introduction}

Inadequate gut absorptive surface area or defective gut motility may lead to total parental nutrition (TPN) dependence, termed intestinal failure (IF). Congenital anatomic abnormalities, necrotizing enterocolitis and severe motility defects account for most cases of IF [1]. Neonates with IF have prolonged admissions in the neonatal intensive care unit (NICU), have increased healthcare expenditures [2], and ultimately require lifelong follow-up with gastroenterology (GE) as outpatients. In most centers, NICU care of these patients is shared between NICU teams, surgeons, and GI consultants who rotate on and off service at least weekly, with variable interest and expertise in IF. Long-term decision making in this complex patient population is thus shared amongst short-term providers who may or may not have an investment in outpatient care, and who may not feel that they have the resources to discharge a patient on parenteral nutrition. In our own $\mathrm{NICU}$, we noticed that this pattern was associated with highly variable discharge criteria and unnecessary increases in length of stay (LOS). Patients were often held to attempt to achieve arbitrary enteral feeding goals when it was clear from their post-operative anatomy that they would never achieve enteral autonomy in the first $6-12$ months of life. Outpatient-based multidisciplinary care models for IF have been described and were associated with improved patient outcomes [3], but little is published on inpatient care programs or optimal discharge timing from the hospital. We aimed to assess the positive and negative impact of a simple intervention in the NICU to shorten LOS for IF patients.

\section{Materials and Methods}

We undertook a quality improvement initiative to centralize 
Table 1. Optimal Discharge Timing Criteria for Neonates With Intestinal Failure

A neonate can be considered for discharge and outpatient gastroenterology follow-up when:

Continuous enteral feedings of at least $5 \mathrm{~mL} / \mathrm{h}$ for $48 \mathrm{~h}$ are tolerated

An indwelling central venous catheter appropriate for home use is in place

Parenteral nutrition is cycled to at least $20 \mathrm{~h}$ /day or less and tolerated

No other medical conditions requiring NICU care are present
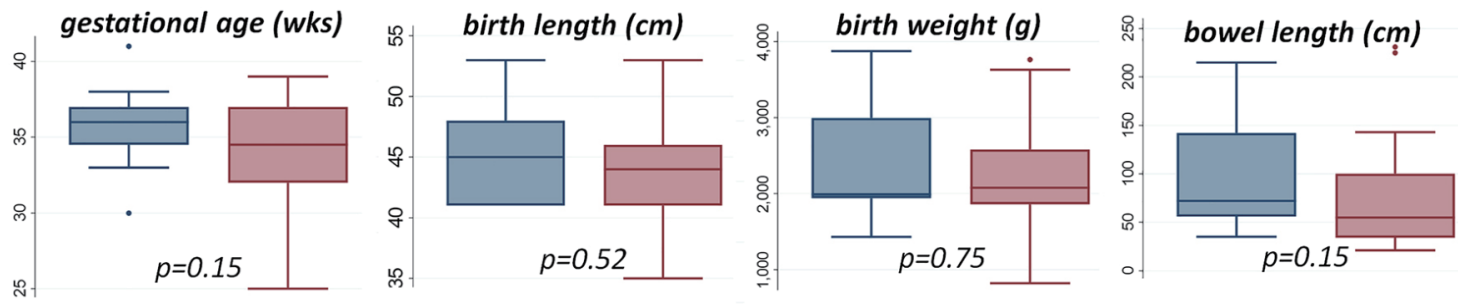

Optimal cohort

Historical cohort

Figure 1. Baseline patient characteristics in the optimal discharge versus historical cohorts.

care in NICU patients with IF and institute standardized discharge criteria. Two gastroenterologists who manage outpatients with IF, central lines and TPN were assigned to rotate weekly in our NICU. They performed all nonurgent consultations, family care conferences, and weekly inpatient followup. We implemented standardized, optimal discharge goals for patients with IF (Table 1) to better identify patients who could be safely cared for at home. All post-NICU outpatient care was performed by the same two gastroenterologists. After 18 months, we reviewed medical records of patients who met optimal discharge criteria and compared their outcomes to a historical cohort of IF patients. We assessed frequency of central line-associated bloodstream infection (CLABSI) as well as readmission to the hospital. This study was conducted in compliance with the ethical standards of the responsible institution on human subjects as well as with the Helsinki Declaration, and with institutional review board approval.

\section{Results}

During the study period, optimal discharge criteria were met in 12 patients with IF (optimal group). Three patients with IF did not meet discharge criteria due to needs surrounding care of a tracheostomy, progressive liver disease, and lack of a safe home environment, respectively. A cohort of 26 historical patients was identified (historical group) for comparison. The etiology of IF in the optimal versus historical group was gastroschisis with bowel atresias in $75 \%$ vs. $53 \%$, volvulus in $8 \%$ vs. $11 \%$, necrotizing enterocolitis in $9 \%$ vs. $27 \%$, and other in $8 \%$ vs. $9 \%$, respectively (all $\mathrm{P}=\mathrm{NS}$ ). Patients in optimal and historical groups had similar baseline characteristics (medians, all $\mathrm{P}=\mathrm{NS}$ ): gestational age (36 vs. 35 weeks), birth weight (1,990 vs. 2,076 g), birth length (45 vs. $44 \mathrm{~cm})$, and small bowel length after definitive surgery (63 vs. $55 \mathrm{~cm})$, re- spectively (Fig. 1). Compared with the historical group, the optimal group was discharged much earlier (median LOS 69 vs. 126 days, $\mathrm{P}<0.01$ ) (Fig. 2), experienced fewer CLABSIs ( 4 vs. 10 per 1,000 patient days, $P=0.04$ ), and was readmitted less frequently ( 7 vs. 17 per 1,000 patient days, $\mathrm{P}<0.01$ ). Both groups achieved enteral autonomy during the first year after discharge at similar rates: 5/12 (42\%) in optimal and 8/26 $(31 \%)$ in historical groups $(\mathrm{P}=\mathrm{NS})$. There were no deaths in 1 year of post-discharge follow-up in either group.

\section{Discussion}

When management of IF in the NICU was restricted to a small team of gastroenterologists with an investment in outpatient

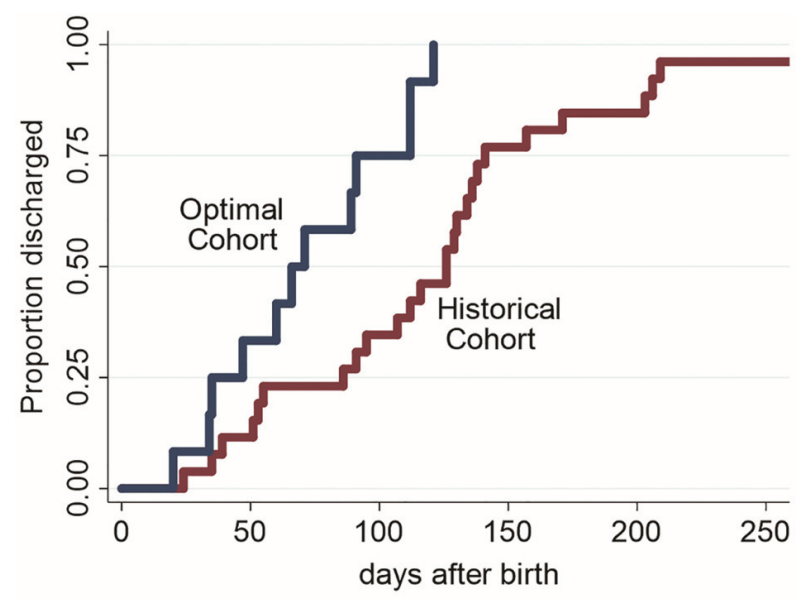

Figure 2. Time to NICU discharge amongst infants with intestinal failure utilizing optimal discharge criteria versus historical management practices. 
care who instituted standardized discharge criteria, LOS decreased substantially without untoward safety events. This change in practice resulted in savings of 684 NICU days (57 days per patient), with fewer CLABSIs after discharge and fewer readmissions compared to historical management practices. At approximately 4,000 dollars per day in hospital charges to stay in a NICU, this program saved over 2.7 million dollars in care costs while allowing families and their infants more time at home. Providers established early relationships with families over multiple encounters prior to discharge, with repeated opportunities for education, anticipatory guidance and shared decision making. Many patients with IF can be managed safely at home, at substantial cost savings. The safety and applicability of the optimal discharge criteria presented here should be studied further. Similar programs may be effective at other large academic medical centers.

\section{Acknowledgments}

None to declare.

\section{Financial Disclosure}

MD is funded by the Primary Children's Hospital Foundation, and the National Center for Advancing Translational Sciences of the National Institutes of Health under Award Numbers KL2TR001065 and 8UL1TR000105 (formerly UL1RR025764). The content is solely the responsibility of the authors and does not necessarily represent the official views of the National Institutes of Health.

\section{Conflict of Interest}

MD has consulted for HighTide Biopharmaceuticals USA. EJ and $\mathrm{AE}$ have nothing to declare.

\section{Informed Consent}

Not applicable.

\section{Author's Contribution}

All authors participated in study design, data collection, data analysis, and manuscript preparation.

\section{References}

1. Salvia G, Guarino A, Terrin G, Cascioli C, Paludetto R, Indrio F, Lega L, et al. Neonatal onset intestinal failure: an Italian Multicenter Study. J Pediatr. 2008;153(5):674676.e2.

2. Kosar C, Steinberg K, de Silva N, Avitzur Y, Wales PW. Cost of ambulatory care for the pediatric intestinal failure patient: One-year follow-up after primary discharge. J Pediatr Surg. 2016;51(5):798-803.

3. Stanger JD, Oliveira C, Blackmore C, Avitzur Y, Wales PW. The impact of multi-disciplinary intestinal rehabilitation programs on the outcome of pediatric patients with intestinal failure: a systematic review and meta-analysis. J Pediatr Surg. 2013;48(5):983-992. 